\title{
MATHEMATICS IN ECONOMICS
}

\author{
WASSILY LEONTIEF
}

1. Gibbs and mathematical economics. American economists have a good and special reason to honor J. Willard Gibbs. The late Professor Irving Fisher - the author of the earliest monograph on Mathematical Economics published on this side of the Atlantic and one of the truly great economists this country has produced-was a pupil of Gibbs. He was in 1929 the first to represent social sciences in this series of memorial lectures. The second was Professor Edwin B. Wilson, mathematician and economist, also one of Gibbs' immediate disciples, and author of the early treatise on Vector Analysis based on his teacher's original lectures on that subject.

Professor Fisher and Professor Wilson were leading spirits in the organization - twenty-three years ago - of the international Econometric Society which now unites 2500 economic statisticians and economists who claim the ability to speak-or at least to understand when spoken to- the "language of mathematics" which Josiah Gibbs used with such compelling and poetic power.

I did not know Gibbs and I am not a mathematician. I cannot present to you personal reminiscences about this great man nor am I able to develop before you any one particular application of mathematics to economics - which could possibly be of technical interest to a professional mathematician. I will try instead to survey the logical structure of the present day economic theory emphasizing formal aspects of some of the problems which it faces and pointing out the mathematical procedures used for their solution. The views to be presented are, of course, not necessarily shared by other economists. Even leaving out those who feel with Lord Keynes that mathematical economics is "mere concoctions," theoretical disagreements and methodological controversies keep us from sinking into the state of complacent unanimity.

2. The general structure of economic theory. The object of economic analysis is the observed, or at least the observable, economic process. The typical variables in terms of which an economic system is described are the amounts of various goods and services produced, consumed, added to and subtracted from existing stocks, sold and

The twenty-seventh Josiah Willard Gibbs Lecture, delivered at Baltimore, Maryland on December 28, 1953, under the auspices of the American Mathematical Society; received by the editors January 11, 1954. 
purchased; also the prices at which these purchases and sales are made.

The available quantities of natural and human resources, the state of technical knowledge and the nature of consumers' preferences (with those, in our modern much regulated economy, one must mention also the aims and preferences of the regulating governmental authorities) - all described within the setting of a specific institutional framework-constitute what might be called the operating conditions of the particular economic system. These are the "data" which in verbal analysis are used to explain the "unknown" outputs, employment, prices, investments, and so on.

Translated into mathematical language this means that the available quantities of natural and human resources, the state of technical knowledge and consumers' preferences determine the structure of equations (or inequalities) which in their turn determine the values taken on by what we choose to define as the dependent "variables" of the economic system.

The first systematically formulated mathematical theory of general Economic Equilibrium was constructed just about seventy-five years ago by Léon Walras [1]. He incorporated in it much of the so-called classical theory developed in the writings of the great English and French economists of the late eighteenth and early nineteenth century. Some essential pieces of the conceptual apparatus used by Walras - such, for example, as the concepts of supply and demand functions and the notion of diminishing marginal utility-were already cast in mathematical form by such men as Daniel Bernoulli [2], Augustin Cournot [3], and E. J. Dupuit [4].

Elaborated and extended by Vilfredo Pareto [5] and his contemporaries and successors, the general theory of economic interdependence is gradually being combined-into what promises to become a unified logical structure - with two other fields of analytical inquiry, the theory of market mechanism and the analysis of the behavior of an individual firm and of a separate household.

3. Maximizing behavior. It is in this latter connection, in explanation of the operation of the ultimate decision-making units, that the common notion of "economic behavior" finds its principal analytic application.

Consider the profit maximizing firm. It purchases or hires certain commodities and services and utilizes them for the production of other commodities or services. The production process itself can be described as a transformation of one set of variables-the inputs, 
into another-the outputs. The quantitative relationships between the inputs and the outputs are determined by the set of all available technological alternatives.

The outlays, the costs, incurred by the firm can obviously be considered as a function of the input combination used, while its gross revenue depends upon the amounts of its outputs. Among all the input-output combinations technically attainable, the firm chooses the one which maximizes the difference between its total costs and revenue.

In a simple case in which all available transformation possibilities are stated in the form of one or more well behaving "production functions" with continuous derivatives throughout the entire relevant range, a local maximum can be described by a set of simple equations involving its first partial derivatives and parameters entering the profit function such, for example, as the prices of all commodities sold and purchased.

It is not surprising that these conditions were discovered and stated by some economists verbally without any recourse to mathematics. A correct formulation and interpretation of the secondary conditions for a maximum, involving inequalities in higher derivatives, had, however, to wait for the introduction into the argument of formal calculus.

The problem becomes more intricate as soon as the well behaving continuous production functions are replaced by the more realistic description of technical input-output relationships involving linearities, discontinuities, and inequalities. Then the question concerning the optimization conditions in the small is replaced by their study in the large. Under the name of "linear programming" much advanced work has, for example, been done recently on the problem of determining maxima with the constraining transformation functions stated in the form of a set of positive vectors; the positive and negative components of each vector describe in this case the sets of outputs and, respectively, inputs corresponding to the operation on a unit level of one particular kind of productive activity. Differential calculus and elementary algebra-the two traditional tools of the mathematical economist - are being thus replaced or at least supplemented by those of topology and matrix algebra.

The explanation of consumer's behavior is developed along similar lines. A household like a firm has an income (derived from the sales of the services of persons or property rights) and an outlay; to the transformation functions of the firm there corresponds the utility 
function of the household. It describes the level of satisfaction corresponding to the amounts of goods and services consumed.

Within the constraints imposed by its budget, the household is supposed to select a combination of goods and services which brings it to the highest level of satisfaction. In early theories, utility was treated as a measurable quantity. On closer examination, its cardinal measurement turned out to be neither necessary for formulation and solution of the maximum problem at hand nor, essentially for that very reason, operational in terms of actual experience.

Consider two individuals facing identical budgetary restrictions. If one of them derives from any combination of commodities consumed, say, twice as much satisfaction as the other, both will obviously find their respective utilities maximized by exactly the same sets of purchases. Insofar as a consumer's observed movements through commodity space constitute the only objective source of information about the shape of his utility function, ordinal comparison of its different levels is all that can be achieved or required for explanatory purposes.

This is where the matter stood till the recently revived interest in the old eighteenth century problem of choice under conditions of uncertainty led to renewed attempts to rehabilitate the cardinal utility function. The argument hinges on the assertion [6] that from the point of view of "rational" behavior, if,

(a) $U\left(X_{1}\right)$ and $U\left(X_{2}\right)$ are the utility levels associated in the mind of a decision-making consumer with certain but alternative possession of the two specific commodity combinations, $X_{1}$ and $X_{2}$, and

(b) $p$ is a true positive fraction such that

(c) this consumer, when offered the choice between the "chance with the probability $p$ of possessing $X_{1}$ " and "the chance with the probability $(1-p)$ of possessing $X_{2}$ " will find both these offers to be equally desirable, then,

$$
\frac{U\left(X_{1}\right)}{U\left(X_{2}\right)}=\frac{(1-p)}{p} .
$$

Once this is admitted, a cardinal comparison of utilities must obviously be accepted as operationally feasible. Whether a particular individual actually behaves in accordance with this assertion or not can be empirically tested - through introduction of a third commodity combination, $X_{3}$, with an accompanying probability, $q$. Two choices, one between the chances involving $U\left(X_{3}\right)$ and $U\left(X_{1}\right)$, and another involving $U\left(X_{3}\right)$ and $U\left(X_{2}\right)$, should lead to measures consistent with the comparison of $U\left(X_{1}\right)$ and $U\left(X_{2}\right)$ as shown above. If 
they do not, the subject of the experiment is declared to be "irrational." The reference to "rational" or, should I say, "economic" behavior as used in this connection is intended to justify the acceptance of a crucial proposition "ex definitione." Substantively, it denies the phenomenon of the pleasure (the utility) of gambling by disallowing the possibility of using a utility function of the more general form, such, for example, as $U\left(X_{1}, p\right)$.

In this, as in many other similar instances, the economist must be prepared to make up his mind whether he is aiming at a positive explanation of observed facts or at setting up normative rules for, in some sense, "reasonable" behavior and tracing out their logical implications.

In the discussion of public economic policies-in contrast to the analysis of individual choice - the normative character of the problem has been clearly and generally recognized. Here the mathematical approach has crystallized the analysis around the axiomatic formulation of the (desirable or conventional) properties of the "social welfare function." Social utility is usually postulated as a function of the ordinally described personal utility levels attained by each of the individual members of the society in question.

The only other property on which something like a general consensus of opinion seems to exist is that "the social welfare is increased whenever at least one of the individual utilities on which it depends is raised while none is reduced." Without any furthermore stringent limitation on its possible shape, such a social welfare function allows only a partial ordering of all possible combinations of individual utility levels. A much more specific description of its properties would have to be required if the social welfare function were to reflect-in axiomatic formulation-concrete normative judgments pertaining, for example, to the problem of income distribution. The struggle to increase the utility levels of some groups of individuals at the cost of reducing the welfare of others constitutes, no doubt, the core of much of the present day politico-economic controversy.

The important contribution of the mathematical approach to our thinking on such controversial issues consists in showing how difficult it actually is to formulate in concise operational terms any specific normative attitude toward questions of public welfare in general and the problem of equitable distribution of income in particular.

4. Consistency criteria in the theory of interdependent choices. The analysis of the behavior of individual firms and households is and-if it has to have explanatory rather than normative significance - should be not more than a direct translation into concise mathe- 
matical language of problems of maximizing choice as seen from the actual decision makers' point of view. The restraining relations and parameters which the economist assumes as "given" must, of course, be precisely those which the household or firm actually considers as being independent of its action, and the set of variables - the optimal combination of which the theorist explains-must indeed include all those, and only those, on which the real economic units actually operate in putting into effect their profit or, respectively, utility maximizing decisions.

So long as one does not radically widen the conventional universe of economic discourse, the invariance of technological transformation functions in respect to changes in specific input combinations can be taken for granted. The same, however, cannot be said about the functions and parameters which-although they are treated as fixed constraints in the explanation of individual maximizing behaviorwithin the larger framework of the general theory of economic interdependence turn up in the role of dependent variables rather than of "given" data.

Farmer Jones, when he decides on the most profitable number of hogs to grow, takes into account the market price at which they can be sold. In doing so, he most likely considers that price as "given," i.e., to be practically independent of the specific outcome of that decision. In explaining farmer Jones's output, the economist accordingly treats the price as one of the parameters entering the solution of the corresponding profit maximization problem.

In his very next step, in presenting the general equilibrium theory (which I will presently take up), the economist lists all prices-including the price of hogs-among the unknowns to be determined through the solution of an appropriate system of equations. In particular, he then proceeds to explain, in terms of that system, why the price of hogs would fall if all farmers, say, for experimental purposes, had produced and thrown on the market 10 per cent more hogs than before. Another argument based on the same general equilibrium equations shows that, within the range of output variations accessible to him, farmer Jones's belief in his own inability to affect the market price of hogs to any appreciable extent is indeed entirely correct. If, however, it had turned out-again within the framework of the general equilibrium theory - that farmer Jones's individual action could have influenced the price of hogs - as indeed would have been the case had he owned half of all the hogs in the country - the entire analysis in both its parts would have been false. The explanation of farmer Jones's maximizing behavior, because it was derived from an as- 
sumption that now proved to be inconsistent with the implication of further general equilibrium analysis based on that very explanation, the general equilibrium analysis, obviously would be false for the same reason.

All problems dealt with in the analysis of market behavior lead to such questions of theoretical consistency. Their logical structure is frequently quite subtle and the circular test outlined above is difficult to apply without recourse to mathematical formulation.

The analysis of duopology and oligopoly, i.e., of the relationships between two or few mutually interdependent sellers, also the explanation of bilateral monopoly, a situation in which a single seller faces a sole buyer, each clearly and appreciably affecting by his actions the others' profit, all lead to the same theoretical problemthe explanation of maximizing behavior of two or more mutually interdependent units.

Beginning with Augustin Cournot [3], that is, for over a century, mathematical economists have wrestled with that question without apparent success. The modern Theory of Games [7] has contributed greatly toward a more concise formulation of the issues involved, but an acceptable theory of interdependent maximizing behavior has yet to be offered. As in the discussion of the cardinal measure of utility, an elaboration of the logical consequences of arbitrary normative assumptions here, too, has occasionally been mistaken for a solution of the positive problem. Possibly, such a solution can be even shown not to exist.

5. The theory of general interdependence. The Theory of General Equilibrium - the analysis of the mutual interdependence of all the producing and consuming units making up a national economy orif one wants to take into consideration international trade-the world economy as a whole, makes up the core of modern economic theory.

The simplest standard model of the general equilibrium systemstripped of all optional equipment and adornments-is designed to explain the determination of the (time-) rates of production (sales) and consumption (purchases) of all commodities and services by each of the individual decision-making units as well as the prices at which all these inputs and outputs are traded.

The explanation is presented in the form of a system of simultaneous equations. Their number just suffices to determine the values -unique or multiple - of the unknowns. All sales and purchases of each particular commodity are supposed to be transacted at the 
same price and the prices of all commodities and services are to be such as to make the combined output (supply) of each commodity by all the units equal to its aggregate input (demand) by all the units.

The quantity of each commodity produced or consumed (it could be both) by any unit has already been shown to depend - through the budgetary restriction - on prices; its own as well as those of the other goods. The "supply" and the "demand" functions, so frequently referred to by the economist, are meant to describe this dependence; their shape is obviously implicitly determined by the equations (or inequalities) which in the description of its maximizing behavior served to determine the optimal position of the individual decisionmaking unit in the commodity space.

Although some of its constituent equations are thus based on the satisfaction of certain maximizing conditions, the general equilibrium system itself cannot legitimately be thought of in any other but quasi-mechanical terms. This does not mean that an eighteenth century believer in the Invisible Hand or his present day counterpart, the modern welfare theorist, could not have legitimate interest in finding out whether the actual economy - as described by the set of the general equilibrium equations-does or does not satisfy the normative social welfare criteria of his particular choice.

Let me add that under certain ideal conditions, the outcome of the automatic operation of the competitive price mechanism, as reflected in the general equilibrium system described above, can be shown-so far as the organization of production is concerned-to be identical with that which would be achieved by an omniscient and all-powerful planning committee of efficiency experts. In a state satisfying the Walrasian equilibrium equations, the total output of no commodity can be increased and the input of no scarce primary resource diminished without reduction in the output of at least one other commodity or an increase in the input of at least one other commodity or an increase in the input of at least one other scarce primary resource.

In other words, if outputs are measured as positive and inputs as negative quantities in the many dimensional commodity space, the actual equilibrium position of a competitive economy is represented by a point located on the hull of the compact space comprising all input-output combinations attainable to it on the basis of the given transformation functions; each vector connecting any two points on that hull necessarily contains components of opposite signs.

This obviously applies to any optimal position which an individual 
profit-maximizing enterprise would choose among all the input-output combinations attainable to it.

The truth of that theorem in the case of the competitively operating economy as a whole follows from the fact that it can be shown to apply to the sum of the optimal input-output vectors of any group of profit-maximizing enterprises simultaneously operating within the same price system.

This makes it possible for the economist, when he studies the quantitative aspects of the input-output relationships within the theoretical framework of a competitive general equilibrium system, to disregard its subdivision among the many individual enterprises and to speak of an "industry," groups of industries, and even of the economy as a whole as if it were a large single enterprise.

6. Dynamics. The quasi-mechanical nature of the economic system as a whole becomes particularly clear when, as has occurred over the last twenty-five years, the mathematical economists engaged increasingly in exploring its dynamic properties.

The static, essentially timeless system of general equilibrium equations described above is an idealization of limited empirical validity. The technical transformation functions, for example, in order to reflect more closely the conditions of actual production, should contain the values of at least some of the variables as related to different points in time: This year's harvest depends on last year's sowing.

Consider, for instance, the process of economic growth. Insofar as it involves the accumulation of capital, its explanation leads back to the fundamental observation that the output of a finished productexpressed as a rate of flow, per unit of time-cannot be described as depending only on the flow rates of requisite inputs. It requires also the presence of certain specific stocks: stocks of buildings, stocks of machinery, inventories of raw materials and of intermediary semifinished products. But stocks can mostly be described as flow rates (or differences of flow rates) integrated over time.

The dynamic process of capital accumulation in its simplest form can be described and explained in ordinary language. With the introduction of other kinds of dynamic relationships, the theoretical system becomes unmanageable without the use of mathematics. The theory of the so-called "business-cycle," that is, of the fairly regular succession of ups and downs in output, employment, trade and prices experienced by all advanced western economies is a case in point. From the time the first major nineteenth century depression hit England in 1819, economists have searched for a systematic ex- 
planation of that phenomenon. But not before the nineteen thirty's when the mathematical economists became interested in the subject was there introduced into its discussion the notion of self-generated periodic fluctuations corresponding to the pairs of complex roots which frequently appear in the solution of difference or differential equations [8].

No wonder that from that time and up to two or three years ago, when the theory of games and problems of linear programming came in vogue, dynamic general equilibrium theory has been the favored hunting ground of mathematically inclined economists. Integral and differential, difference and mixed difference and differential equations, phase graphs of linear and nonlinear oscillating systems-all of these and many other tools of applied analysis have found their place in the recent discussion of economic dynamics. Having likened the austere outlines of Walras's original general equilibrium system to a standard, stripped down "model T," I cannot help but compare some of the latest dynamic models with the super-deluxe editions of hard top convertibles equipped with everything from white-wall tires to a concealed bar.

7. Paucity of operationally significant conclusions. One has, unfortunately, to admit that neither the simpler type of economic theory nor its most modern dynamic versions have brought us very far along the road toward detailed explanation, not to say prediction, of the specific states of the actually observed economic system.

Seldom, in modern positive science, has so elaborate a theoretical structure been erected on so narrow and shallow a factual foundation. Traditionally-and that tradition still prevails among mathematical and nonmathematical economists alike- "pure" theory has not been implemented with empirical determination of any of the numerical parameters involved. As can be seen even from the sketchy outlines presented above, all empirical assumptions on which such theories are based are qualitative in character and, at that, they are quite vague and general. So are the few operational propositions at which pure economic theory arrives.

Paul Samuelson, who more than anybody else contributed to the systematic codification of modern economic theory and a clarification of its logical structure [9] pointedly brings out the parallelism between the method used by economists to derive certain meaningful implications of maximizing behavior and the elegantly general modes of reasoning found in J. Willard Gibbs' celebrated treatise On the equilibrium of heterogeneous substances. The following simple but 
typical argument from the theory of consumers' behavior will show what I have in mind.

Let the elements of a non-negative row matrix, $X$, represent the quantities of commodities which a household, with a dollar income, $r$, can purchase at prices described by the element of the column matrix, $P$. Under the budgetary constraint,

$$
X P_{i}=r_{i},
$$

where the subscript, $i$, is used to identify some specific price income situation, the household will choose to purchase those particular amounts, $X_{i}$, which will maximize his utility $U(X)$. About $U(X)$, we only know (a) that it is a nondecreasing function of $X$ and (b) that-since utilities can be compared only in the ordinal sense-it admits transformation by any increasing function, $F(U(X))$.

Let $X_{1}$ and $X_{2}$ represent the optimal consumption patterns corresponding to two different price-income situations $P_{1}, r_{1}$ and $P_{2}, r_{2}$. If

$$
X_{1} P_{1}=r_{1} \geqq X_{2} P_{1}, \text { then necessarily } U\left(X_{1}\right)>U\left(X_{2}\right),
$$

since otherwise when placed in the price-income situation $P_{1}, r_{1}$, the consumer would purchase $X_{2}$ rather than $X_{1}$.

For analogous reasons,

$$
U\left(X_{1}\right)>U\left(X_{2}\right) \text { implies } X_{2} P_{2}=r_{2}<X_{1} P_{2} .
$$

It follows that

$$
\left(X_{1}-X_{2}\right) P_{1} \geqq 0 \text { implies }\left(X_{1}-X_{2}\right) P_{2}>0 .
$$

Propositions (2) and (3) make it possible in some, but unfortunately not in all, cases to infer from the change in the observed pricepurchase pattern of the consumer to the direction of the corresponding change in his level of welfare. Proposition (4) imposes certain limitations on the shape of individual demand functions.

Analogous arguments make it possible to impose similar empirical limitations on the shape of the behavior equations of profit maximizing firms.

Insofar as the individual demand and supply functions enter into the analysis of the economy as a whole, these limitations carry over into the general equilibrium system as well. This applies, in particular, to the input-output relationships characterizing the operations of the productive sectors of the economy. As mentioned before (see p. 223), whenever the economy operates within the framework of competitive pricing, these relationships are identical with 
those which would have prevailed within one single large profit maximizing enterprise operating on the basis of the same technological horizon. This is why the "pure" general equilibrium theory seems to yield richer empirical results in respect to production than when it deals with household consumption. It also explains why in studying the quantitative aspects of an economic system one sometimes legitimately disregards the details, or should I say accidents, of its particular institutional organization and conducts the entire analysis in terms of only such basic data as the supply of primary resources and the "state of the arts," i.e., the technologically given transformation functions. Reduced to these simplest terms, the same general theoretical propositions apply to the highly advanced American private enterprise economy, the centrally planned Soviet system and, say, the economy of an isolated primitive tribe.

With all that, or rather because of that, the legitimately harvested empirical yield of the general equilibrium theory is very limited. An interesting attempt was made by Abraham Wald [10] to impose further limitation on the admissible shape of the general equilibrium equations by introducing the requirement that all prices and quantities as determined by it have to be positive. On closer examination the operational implications of this argument turn out to be disappointing: Any number of alternative sets of sufficient conditions for such a result can be stated, but they obviously would be of little significance from the economic point of view. The necessary conditions for such a positive solution applying to any particular observed price output situation would, on the other hand, be so special that even if stated explicitly they also would be devoid of empirical interest.

Furthermore, the entire question is misplaced from the point of view of the purpose it is intended to serve. One of the interesting empirical questions which an economist occasionally has to answer is whether with a given combination of (virtual) operating conditions an economic system would be capable of yielding positive outputs, i.e., whether it could exist at all. In posing the question, one obviously must allow for the possibility of a negative answer.

In dynamic analysis, a similar search for additional limitations on the empirically admissible shapes of the basic quantitative relationships has produced the suggestion that only convergent systems leading to stable solutions should be considered. If that proposal were taken seriously, how would we go about explaining the rapid and apparently limitless growth of the modern western economies? 
8. Indirect inference. Please note that while questioning the validity of such quasi-empirical generalization, I do not impugn the error of circular reasoning to those who make them. If the invisible, but indirectly inferred structural properties of a theoretical system were used to "explain" only those of its directly observed characteristics from which these properties were derived in the first place, the argument could indeed be rejected as heuristically useless. When, however, the indirectly inferred structural properties of the theoretical system serve to derive new factual propositions, not obviously and immediately related to the first set of empirical statements on which the original inference has been based, the explanatory power of the theory has been clearly increased.

If indirect statistical inference is to be used to obtain the numerical parameters for our system, methods of modern mathematical statistics should obviously supply the tools for such an undertaking. In his 1929 Gibbs Lecture, Irving Fisher refers to "smoothing of statistical time series, correlation and probabilities" as, at that time, newly introduced applications of mathematics to economics. The original systematic attempt to derive a "statistical demand curve" for an individual commodity was made by Henry Moore as early as 1917 [11]. Soon it was followed by a whole series of similar studies.

The first, let me call it the "unsophisticated" phase, of econometric work, aimed at an indirect determination of the structural parameters of the economic system through statistical analysis of the behavior of its variables, reached its high point in the works of Henry Schultz [12], Paul Douglas [13], and Jan Tinbergen [14].

It was characterized by rather careful collection and organization of primary statistical material and straight forward-some might say indiscriminate-application of the "least square" curve fitting techniques in calculation of the actual parameters. There was little in it of mathematical interest (except for Tinbergen's use of difference equations for empirical determination of the complex components of fluctuating time series) and the empirical results obtained appeared to be of rather doubtful significance. After fitting a first, second, or third degree parabola to a price-quantity scatter, one did not know whether it represented the supply or the demand curve for the commodity in question or possibly some weighted average of the two.

It was only natural to make the crudity of the statistical procedure used responsible for such disappointing substantive results. The late thirties and the forties witnessed an unprecedented concentration of 
intellectual effort on problems of methodology [15]. Far from being satisfied with the simple adaptation of recent advances in mathematical statistics, the new econometric school made a number of original contributions to the theory of stochastic systems and the methodology of indirect statistical inference. Such significant advances as the theory of identification (analyzing the relationship between the statistical parameters of an observed stochastic system, on the one hand, and the constants of the underlying theoretical model, on the other) can rightfully be said to have issued from that series of methodological investigations.

The theory of probability and many other modes of mathematical reasoning found in all these studies a most varied and fertile application. I will not invite you now, however, to consider them; they belong in the field of general statistics rather than that of economics. Moreover, the positive contribution which these advanced methods were able to make toward actual empirical determination of the specific quantitative properties of the observed economic system proved to be quite limited, hardly greater than that made by the primitive methods used in studies belonging to the first, unsophisticated phase of that econometric movement.

The explanation of this disappointing result lies, I think, in the fact that for a study of a set of quantitative interrelationships as complex as those underlying a modern economy indirect statistical inference, however refined methodologically, simply will not do [16]. Statistical reliability measures, if properly applied to even the most favorable situation - so far as primary information is concernedyield confidence limits so far apart as to negate the empirical usefulness of the numerical parameters obtained.

It is as if we were asked to reproduce the blueprint of a complicated motor on the basis of our knowledge of the general principles of operation of internal combustion engines and no other specific information but that conveyed by the few dials located on the dashboard and possibly the noise coming from under the closed hood. And as if that were not difficult enough, the structural characteristics of the engine the economist is studying are known to change under the impact of its continual operation.

The task as presented can hardly be accomplished. It certainly becomes much easier if we are allowed to look under the hood. It would, of course, be even more convenient if it were possible to stop the motor, take it apart and subject each of its components to any desired tests and measurements. That is what experimental scientists can do and economists cannot. But look under the hood he can, al- 
though in economics as in the garage it is an inconvenient and often a dirty operation. Admittedly, had we been able to reproduce the blueprint of the engine by indirect inference from the behavior of the gauges, such intellectual accomplishment would earn a much higher rating. Nevertheless, some economists rolled up their sleeves and looked under the hood.

9. Direct structural analysis. Direct observation and detailed description of the various aspects of economic reality are as old as our discipline itself. As statistical information became more and more available, descriptive quantitative economics came to be recognized as one of its important branches. But far from seeking to establish a close - not to say intimate-cooperation with the theorist, many of the empirically minded investigators came to consider direct observation as a separate self-sufficient approach to the explanation of the functioning of the economic system rather than the necessary descriptive complement of its theoretical analysis.

The empiricist school developed even a kind of a quantitative descriptive technique all its own. Its principal or rather only tools are averaging and aggregation (an aggregate of a set of magnitudes is defined as a weighted sum of its elements). While the theorist discovers or, should I say, introduces order into the complex multiplicity of measurable economic phenomena by reducing it to a system of equations, the radical empiricist simplifies the quantitative picture by describing it in terms of a few broad aggregates and averages. A detailed tabulation of the amounts of all the many kinds of goods and services consumed in the course of a given year by households or invested in all the various industries to expand their productive capacity is replaced, for example, by one single figure identified as the annual Net National Income. This figure represents the sum total of the dollar values of all the individual commodities and services mentioned above. Similarly, the multidimensional set of the corresponding prices is replaced by a single number-a weighted average of its individual components-called "the General Price Level."

As conveniently simplified-but albeit necessarily blurred-descriptions of quantitative phenomena such aggregates and averages proved to be useful, nay, indispensable to economists. Even pure theorists use it - more of ten probably than they should-as a pedagogical device which lends the appearance of realism to their schematic general equilibrium models. Some of these models purport to depict the operation of the entire economic system in terms of five, four, or even only three aggregative variables. As a substitute for 
theoretical analysis and generalization, such simplifying devices are obviously valueless. To the extent to which broad aggregates are not directly observable (and few of them are) but have to be compiled from separate measurements of the component variables, in utilization of primary observational data, no economy can be achieved through their use either.

Direct factual study and quantitative descriptions of the structural properties of the economic system, detailed in content, comprehensive in coverage, and systematically designed to fill the specific requirement of an appropriate theoretical scheme, seem to offer the only promising approach to empirically significant understanding of the operational characteristics of the modern economy.

The task thus imposed on the collector of primary factual information exceeds by far anything demanded hitherto from quantitative empirical research, in economics or any other social science. It is only reasonable to suggest that the theorist should meet him half way by redesigning his analytical scheme so as to take advantage of the strengths and mitigate the weaknesses of the observational data to which it will have to be applied.

As an example of such mutual accommodation between theoretical formulation and observational capability, let me say a few words about the so-called input-output analysis in which I myself happen to have an interest of long standing, [17] and [18].

The difficulty, the challenge which an economist faces in trying to analyze and to describe in concrete numerical terms the specific operational characteristics of a modern national economy is caused by the complexity of the interindustrial or, more generally, intersectoral relationships which it comprises. A reduction in the consumers' purchases of automobiles leads, for example, to a fall in the demand for the electric energy required for production of aluminum which goes into the manufacture of cylinder heads.

Economic theory tells us that in order to trace through such a chain of relationships, one must determine the actual shape of the transformation (production) functions of all the individual sectors of the economy in question, insert them into an appropriate system of general equilibrium equations and finally compute the effect which the assumed increase or decrease in final demand would have on the output in question.

Since a fully detailed description of the actual shapes of all the transformation functions comprised, say, in the structure of the American economy, is obviously impossible, the theory had to be reformulated in terms of linear transformation functions-considered 
to represent the first approximation to the actual functions. The solution of the general equilibrium problem was accordingly reduced to inversion of the coefficient matrix of a system of linear equations: If $a_{i k}$ represents the input coefficient showing the number of units of the product of industry $i$ absorbed by industry $k$ per unit of its respective output, the relationships between the total gross outputs, $x_{1}, x_{2}, \cdots, x_{n}$, of the $n$ industries constituting the national economy and the so-called final demand comprising consumption and new investment (accumulation), must satisfy the following matrix equation:

$$
(I-A) X=Y \quad \text { or } \quad X=(I-A)^{-1} Y
$$

in which $A$ is the square non-negative matrix of all input coefficients (with $a_{i k}$ its general element), $X$ a column matrix of the $n$ gross outputs, and $Y$ the corresponding matrix of final demand.

Matrix $A$ represents a concise numerical description of the structural properties of the specific economy; it summarizes the results of painstaking and systematic empirical inquiries. Even a highly aggregated picture of the U.S. economy described in terms of $100 \times 100$ matrix required over a year's factual research by 20 trained economists; a more detailed $200 \times 200$ matrix-two years' work by 75 persons. The most detailed input-output matrix of the American economy yet compiled is of the order $450 \times 450$.

The inversion of such matrices presents a real challenge even to modern large-scale computing machines. A system of so many simultaneous linear equations would-if its coefficients were randomly chosen-be highly unstable; its numerical solution would show hardly more than an accumulation of round-off errors. As an economist, I was not astonished, however, to find the inverses of the empirical input-output matrices to be very stable. The economy of the United States actually operates as a kind of a large-scale calculating machine, continuously working out the solution of problems which it poses itself. Applying the conventional standards of computational accuracy, one must say that these solutions certainly do not prove to be excessively unstable.

This last analogy leads quite naturally to the consideration of a workable empirical approach to dynamic problems. Again, the requirements of factual implementation demand the use of a greatly simplified theoretical framework. The dynamic input-output theory which is now undergoing its first empirical tests is based on the introduction into the original static scheme of so-called stock-flow relationships. It leads to the following system of linear differential equations with constant coefficients, 


$$
(I-A) X(t)-B \dot{X}(t)=Y(t) .
$$

The general term $b_{\text {in }}$ of the square matrix $B$ represents the stock of the products of industry $i$ required by industry $k$ per unit of its respective output: $B \dot{X}$ describes the time rate of change of all stocks, i.e., the rate of accumulation or decumulation of all kinds of "capital" in their dependence on changes in the rate of output, $\dot{X}$, of all the individual industries.

The determination of the magnitude of the elements of the capital coefficient matrix $B$ involved a series of empirical studies even more exacting than those aimed at the derivation of the flow coefficients $a_{i k}$.

A $100 \times 100 B$ (stock) matrix of the American economy is now available. A numerical general solution of a homogeneous system of 20 linear differential equations based on consolidated $20 \times 20 A$ and $B$ matrices of the American economy was completed a few weeks ago by Kenneth Iverson on the new Harvard Mark IV calculating machine.

Even if I had time - which I do not have - to outline the study of the formal properties of the linear system described above, and had shown how more and better empirical data will permit the use of a more refined analytical scheme, I admittedly could not dispel the sense of intellectual retreat which by now you must have felt. From the heights of general theorems describing the formal properties of broadly defined systems, we have step by step descended into the realm of elaborate factual observation followed by equally laborious computations: From Gibbs to crude numerical analysis. But such a rebound has probably been unavoidable. Economics, mathematical economics, in particular, acquired very early in its development the attitudes and manners of the exact empirical sciences without really having gone through the hard school of direct, detailed factual inquiry. Possibly it will do us good to be sent down in order that we may catch up with the experience we have missed. And when one has put a hand to it, one cannot help but derive a peculiar satisfaction from seeing masses of seemingly amorphous facts do the bidding of the orderly and ordering mathematical thought.

\section{BIBLIOGRAPHY}

1. Léon Walras, Eléments d'économie politique pure, Lausanne, 1874.

2. Daniel Bernouilli, Specimen theoriae novae de mensura sortis, Commentarii academiae scientiarum imperialis Petropolitanae vol. 5 (1738) pp. 175-92, St. Petersburgh.

3. Augustin Cournot, Researches into the mathematical principles of the theory of wealth (translated from the original, 1838, French ed.), New York, 1897. 
4. Robert Dorfman, Application of linear programming to the theory of the firm, Berkeley, 1951.

5. Vilfredo Pareto, Manuel d'économie politique, Paris, 1909.

6. Jacob Marschak, Rational behavior, uncertain prospects, and measurable utility, Econometrica vol. 18 (1950) pp. 111-141.

7. John von Neumann and Oskar Morgenstern, Theory of games and economic behavior, 2d ed., Princeton, 1947.

8. Ragnar Frisch, Propagation problems and impulse problems in dynamic economics, in "Economic Essays in honor of Gustav Cassel," London, 1933.

9. Paul Samuelson, Foundations of economic analysis, Cambridge, 1947.

10. Abraham Wald, On some systems of equations of mathematical economics, Econometrica vol. 19 (1951) pp. 368-403.

11. Henry L. Moore, Forecasting the yield and price of cotton, New York, 1917.

12. Henry Schultz, The theory and measurement of demand, Chicago, 1938.

13. Paul H. Douglas, Real wages in the United States, 1890-1926, Boston, 1930.

14. Jan Tinbergen, Statistical testing of business cycles theories, II: Business cycles in the United States of America, 1919-1939, Geneva, 1939.

15. Tjalling Koopmans, ed., Statistical inference in dynamic economic models, by Cowles Commission research staff members and guests, Wiley, New York, 1950.

16. Warren Weaver, Science and complexity, American Scientist vol. 36 (1948) pp. 536-544.

17. Wassily Leontief, The structure of the American economy, 1919-1939, 2d ed., New York, 1951.

18. Wassily Leontief et al, Studies in the structure of the American economy, New York, 1953.

HARVARD UNIVERSITY 